\title{
Influence of Departures from LTE on Oxygen Abundance Determination in the Atmospheres of $A-K$ stars
}

\author{
Tatyana Sitnova ${ }^{1}$, Lyudmila Mashonkina ${ }^{1}$, Gang Zhao $^{2}$, \\ Tatiana Ryabchikova ${ }^{1}$, Yury Pakhomov ${ }^{1}$ \\ ${ }^{1}$ Institute of Astronomy, Russian Academy of Sciences, Moscow 119017, Russia \\ email: sitnova@inasan.ru \\ ${ }^{2}$ National Astronomical Observatories, Chinese Academy of Sciences, Beijing 100012, China
}

\begin{abstract}
Solar oxygen abundance is a key parameter for the studies of solar physics. Oxygen abundances of cool stars with different metallicities are important for understanding the galactic chemical evolution. We present non-LTE calculations for O I with the classical plane-parallel (1D) model atmospheres for a set of stellar parameters corresponding to stars of spectral types from A to K. Non-LTE leads to strengthening the O I lines, and the difference between the nonLTE and LTE abundances (non-LTE correction) is negative. The departures from LTE grow toward higher effective temperature and lower surface gravity. In the entire temperature range and $\log \mathrm{g}=4$, the non-LTE correction does not exceed 0.05 dex in absolute value for lines of O I in the visible spectral range. The non-LTE corrections are significantly larger for the infrared O I 7771-5, $8446 \AA$ lines and reach an order of magnitude for A-type stars. To differentiate the effects of inelastic collisions with electrons and neutral hydrogen atoms on the statistical equilibrium (SE) of O I, we derived the oxygen abundance for the five well studied A-type stars. For each star, non-LTE largely removes the difference between the infrared and visible lines found in LTE. In the case of cool stars (Sun and Procyon), inelastic collisions with H I affect the $\mathrm{SE}$ of O I, and agreement between the abundances from different lines is achieved when using the Drawin's formalism for collisional rates calculations. The solar mean oxygen abundance from the six lines is $\log \varepsilon=8.74 \pm 0.05$, when using the MAFAGS-OS solar model atmosphere and $\log \varepsilon=8.78 \pm 0.03$, when applying the 3D corrections taken from the literature. The non-LTE abundances of oxygen are derived for the sample of cool dwarfs with various metallicities on high-resolution spectra observed in the Lick observatory.
\end{abstract}

Keywords. Atomic data, line formation under nonequilibrium conditions, stellar atmospheres, stellar and solar oxygen abundances.

\section{Introduction}

This work is carried out within the joined Sino-Russian project "A systematic study of non-LTE abundances of nearby dwarfs". Its aim is to accurate determination of stellar parameters and abundances of seventeen elements from $\mathrm{Li}$ to Eu taking into account non-LTE effects, (see Chen et al. 2014). The eighty dwarfs with $4600 \mathrm{~K}<\mathrm{T}_{\text {eff }}<6600 \mathrm{~K}$, $-3.0<[\mathrm{Fe} / \mathrm{H}]<0.5$ were selected for this project. High-resolution spectra $(R \simeq 40000)$ with $S / N \simeq 200$ were obtained in Lick observatory, at $3.0 \mathrm{~m}$ telescope with the Hamilton spectrograph.

Oxygen abundances of cool stars with different metallicities are important for understanding the galactic chemical evolution. The O I IR lines at 7771-5 $\AA$ and $8446 \AA$ can be observed in a wide range of spectral types from $\mathrm{B}$ to $\mathrm{K}$, and this is the only set of atomic oxygen lines that is well observed in the spectra of metal-poor stars. It is known that the IR lines have large deviations from LTE and give systematically higher LTE-abundance 


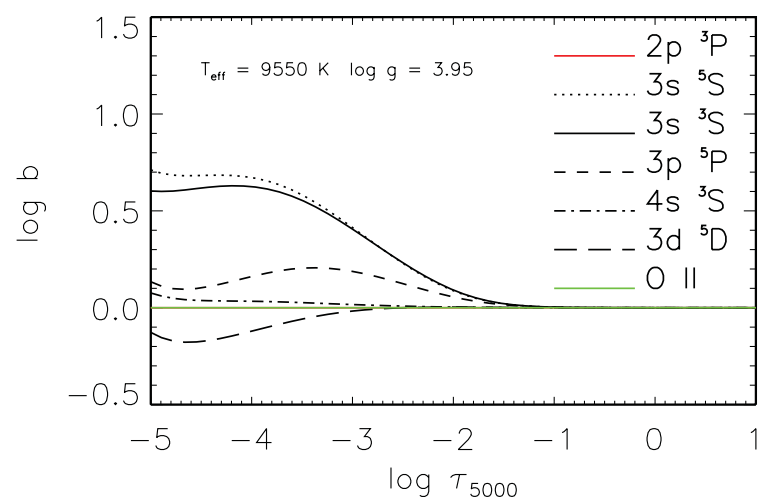

Figure 1. Departure coefficients for oxygen levels in the atmosphere of Vega. The IR 7771-5 lines form in the $3 s^{5} S-3 p{ }^{5} P$ transition. The ground state of $\mathrm{O}$ I is $2 p{ }^{3} P$.

in comparison to $\mathrm{O}$ I lines situated in visual spectral region. For example for Vega $\left(T_{\text {eff }}=\right.$ $9550 \mathrm{~K}, \log \mathrm{g}=3.95,[\mathrm{Fe} / \mathrm{H}]=-0.5)$ the difference in LTE-abundance between the IR and visual lines is $\Delta_{I R-V i s}=\log \varepsilon_{I R}-\log \varepsilon_{V i s}=1.23$ dex. In non-LTE, the corresponding difference is still significant. It amounts to 0.33 dex, when using the $\mathrm{O}$ I model atom from Przybilla et al. (2000). In spite of the non-LTE correction for the IR lines is about -0.9 dex, the deviations from LTE seem to be even larger. We updated the model atom by including the latest data on electron collisions from Barklem (2007) with the hope to remove this discrepancy.

Deviations from LTE are characterized by the departure coefficients $b_{i}=n_{i \mathrm{NLTE}} / n_{i \mathrm{LTE}}$ (Fig. 1). Here, $\mathrm{n}_{i \text { NLTE }}$ and $\mathrm{n}_{i \text { LTE }}$ are the non-LTE and LTE populations of the $i$-th level. Mechanisms of departures from LTE for O I were explained in detail by Sitnova et al. (2013). Here, we describe the non-LTE effects for the O I 7771-5 $\AA$ lines that arise in the $3 s^{5} S^{\circ}-3 p{ }^{5} P$ transition. Both lower and upper levels of this transition are overpopulated relative to their $\mathrm{TE}$ populations in the line formation layers, $\log \left(\tau_{5000} \simeq-2\right)$. However, the lower level is more overpopulated than the upper one, with $b_{3 p{ }^{5} P} / b_{3 s}{ }^{5} S^{\circ}<1$. In non-LTE, the line is affected by the deviation in the source function $\left(S_{\nu}\right)$ from the Planck function $\left(\mathrm{B}_{\nu}\right)$ and the change in opacity $\left(\chi_{\nu}\right)$. These quantities depend on the departure coefficients as follows:

$$
S_{\nu} \sim B_{\nu} b_{j} / b_{i}, \quad \chi_{\nu} \sim b_{i}
$$

As a result, the O I 7771-5 $\AA$ lines are strengthened compared to their LTE strength. The magnitude of deviation from LTE grows toward higher $T_{\text {eff }}$, lower log $g$ and $[\mathrm{Fe} / \mathrm{H}]$, though the behavior of departure coefficients is qualitatively similar for different stellar parameters. The visible O I 3947, 4368, 5330, 6155-9, 6453-4, $7001 \AA$ lines are also strengthened in non-LTE. However, deviations from LTE do not lead to such a dramatic change in the energy absorbed in these lines since they are weak and originate from deep layers. The forbidden [O I] $6300 \AA$ line is immune to departures from LTE.

\section{Non-LTE oxygen abundance determination}

The codes and model atmospheres. We calculated the LTE and non-LTE level populations using the DETAIL code developed by Butler and Giddings (1985). For synthetic spectra calculations we used the SIU (Spectrum Investigation Utility) code developed by Reetz (1999) and synthV-NLTE by V. Tsymbal (private communication). 
We used 1D model atmospheres computed with the following codes: MAFAGS-OS (Grupp et al. 2009) for the Sun and Procyon; LLmodels (Shulyak et al. 2004) for HD 32115, HD 73666, HD17081, and Vega; ATLAS12 (R. Kurucz) for Sirius; MARCS (Gustafsson et al. 2008) for cool dwarfs. Using different model atmospheres does not affect our conclusions, because our aim is to achieve agreement between abundances from different lines in each individual star, but not to compare abundances of different stars.

Testing the O I model atom with hot stars. First of all, we applied the updated oxygen atom model to stars with $T_{\text {eff }}>7250 K$, where there are no uncertainties connected with poorly known inelastic collisions with hydrogen atoms. We selected the five A-type stars with reliably determined parameters to check how the new data for collisions with electrons influence deviations from LTE. Adopted stellar parameters are listed in Table 1. For sources of stellar parameters, observations, and atomic data for the investigated lines see Sitnova et al. (2013). Exceptions are HD 73666 and HD 17081 for which the non-LTE results are presented in this study for the first time. The obtained non-LTE abundances and non-LTE corrections are presented in Table 2. The updated model atom leads to larger deviations from LTE and better agreement of abundances from different lines compared with that for the model atom of Przybilla et al. (2000). For Vega, the difference $\Delta_{I R-V i s}$ did not vanish, but it decreased down to 0.14 dex. For HD 32115 the corresponding difference changes from 0.20 dex (obtained in Sitnova et al. 2013) to 0.09 dex when using new data for electron collisions.

Table 1. Stellar parameters and observations for the reference stars.

\begin{tabular}{llcrrr}
\hline Star & HD & $\mathrm{T}_{\text {eff }}$ & $\log g$ & {$[\mathrm{Fe} / \mathrm{H}]$} & \multicolumn{1}{c}{$\xi_{\mathrm{t}}$} \\
\hline Sun & & 5777 & 4.44 & 0.0 & 0.9 \\
Procyon & 61421 & 6590 & 4.00 & 0.0 & 1.8 \\
& 32115 & 7250 & 4.20 & 0.0 & 2.3 \\
& $73666^{F 07}$ & 9382 & 3.78 & 0.15 & 1.9 \\
Vega & 172167 & 9550 & 3.95 & -0.5 & 2.0 \\
Sirius & 48915 & 9850 & 4.30 & 0.4 & 1.8 \\
& $17081^{F 09}$ & 12800 & 3.75 & 0.0 & 1.0 \\
\hline
\end{tabular}

F07 = Fossati et al. (2007), F09 = Fossati et al. (2009)

Table 2. Non-LTE oxygen abundances of the reference stars.

\begin{tabular}{lccccccc}
\hline Star, HD & $\log \varepsilon_{I R}$ & $\sigma_{I R}$ & $\Delta_{7771}$ & $\log \varepsilon_{V i s}$ & $\sigma_{V i s}$ & $\Delta_{6158}$ & $\Delta_{I R-V i s}$ \\
\hline Procyon, $S_{\mathrm{H}}=0$ & 8.58 & 0.02 & -0.69 & 8.71 & 0.07 & -0.07 & -0.13 \\
Procyon, $S_{\mathrm{H}}=1$ & 8.73 & 0.06 & -0.52 & 8.73 & 0.07 & -0.05 & 0.00 \\
32115 & 8.85 & 0.12 & -0.64 & 8.76 & 0.03 & -0.04 & 0.09 \\
73666 & 8.97 & 0.04 & -1.15 & 8.83 & 0.02 & -0.12 & 0.14 \\
Vega & 8.74 & 0.01 & -0.09 & 8.60 & 0.02 & -0.04 & 0.14 \\
Sirius & 8.57 & 0.04 & -0.82 & 8.43 & 0.03 & -0.02 & 0.14 \\
17081 & 8.77 & 0.03 & -1.50 & 8.75 & 0.03 & -0.18 & 0.02 \\
\hline
\end{tabular}

Solar oxygen abundance. In the atmospheres of cool stars, collisions with hydrogen atoms are more efficient, than collisions with electrons. For hydrogen collision rate calculations we use the Drawin $(1968,1969)$ formalism. An accuracy of this formula is an order of magnitude, so we have to use a scaling factor $S_{\mathrm{H}}$. Fig. 2 shows the solar profile 


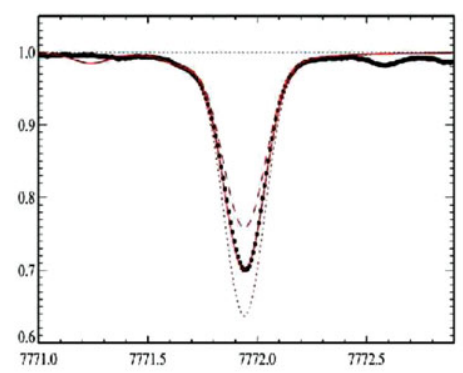

Figure 2. O I $7771 \AA$ in the solar flux spectrum (circles). Solid curve corresponds to non-LTE with $S_{\mathrm{H}}=1$, dotted line to non-LTE with $S_{\mathrm{H}}=0$ (neglecting collisions with hydrogen atoms), dashed line to LTE. All the theoretical spectra were calculated with the same oxygen abundance $\log \varepsilon=8.74$.

of O I $7771 \AA$, LTE and non-LTE synthetic spectra calculated with $S_{\mathrm{H}}=1$ and $S_{\mathrm{H}}=0$ (pure electronic collisions).

The solar oxygen abundances were derived from the visible O I $6300,6158 \AA$ and the IR O I 7771-5, $8446 \AA$ lines. As can be seen from Table 3, there is no agreement between different visible lines: the difference between $6300 \AA$ and $6158 \AA$ is 0.17 dex and 0.15 dex in LTE and non-LTE, respectively. We suppose the abundance from $6300 \AA$ is less reliable due to the uncertainties in continuum normalization because O I $6300 \AA$ is weak and due to blending by Ni I $6300.336 \AA$ line. Also, these lines may be affected by 3Deffects. When applying the (3D - 1D) abundance correction from Caffau et al. (2008), the difference between two visible lines reduces down to 0.07 dex.

In LTE, the abundance from the IR lines is higher than that from the visible ones by 0.14 dex. In non-LTE, if neglecting collisions with hydrogen atoms, abundance from the IR lines turns out even lower than that from the visible lines, with the difference $\Delta_{I R-v i s}=-0.13$ dex. When taking into account $\mathrm{H}$ I collisions, this difference completely vanishes, and solar oxygen abundance is $\log \varepsilon=8.74 \pm 0.05$. When applying the (3D - 1D) abundance correction from Caffau et al. (2008), $\Delta_{I R-v i s}=0.02$ and $\log \varepsilon_{\odot}=8.78 \pm 0.03$.

For comparison with other studies we selected the six common lines of O I and used original model atom from Przybilla et al. (2000). We found well agreement within 0.02 dex between our results and those from Caffau et al. (2008) and Asplund et al. (2004), with $\log \varepsilon_{L T E}=8.85 \pm 0.10 ; 8.86 \pm 0.17 ; 8.87 \pm 0.09$, respectively, and $\log \varepsilon_{N L T E}=$ $8.71 \pm 0.06 ; 8.71 \pm 0.05 ; 8.72 \pm 0.03$, respectively, if $S_{\mathrm{H}}=0$.

From analyses of the solar oxygen lines only we can not constrain $S_{\mathrm{H}}$ solidly. We present non-LTE calculations for Procyon (Table 2). For the weak visible lines in Procyon, $\Delta_{n o n-L T E}$ does not exceed 0.07 dex and for the IR lines, $\Delta_{n o n-L T E}>0.30$ dex in absolute value. The abundances from the two groups of lines coincide when $S_{\mathrm{H}}=1$, and the difference between them is 0.13 dex when $S_{\mathrm{H}}=0$. We can conclude that collisions with hydrogen atoms should be taken into account with $S_{\mathrm{H}}=1$. This is in line with Allende Prieto et al. (2004) who analysed the center-to-limb variation of the O I lines.

Oxygen abundances for the sample of cool dwarfs. From the whole stellar sample we selected the eighteen stars with the best observed spectra. We used stellar parameters listed in Table 4. To determine oxygen abundances, the 7771-5 $\AA$ lines were used for all stars and for the seven stars we also used lines in the visual spectral region. For the latter stars we give $\Delta_{I R-V i s}$ in the last column of Table 4 . For five of them with $[\mathrm{Fe} / \mathrm{H}]$ from -0.71 to $0.00 \Delta_{I R-V i s}$ does not exceed $0.06 \mathrm{dex}$, while for O I $7771 \AA$ non-LTE correction can be up to $-0.41 \mathrm{dex}$. This also supports our choice of $S_{\mathrm{H}}=1$. 
Table 3. Solar oxygen abundance.

\begin{tabular}{|c|c|c|c|c|c|c|}
\hline $\begin{array}{l}\lambda \\
\AA\end{array}$ & $\log \varepsilon_{L T E}$ & $\log \varepsilon_{N L T E}$ & $\Delta_{N L T E}$ & $\begin{array}{c}\log \varepsilon_{N L T E} \\
\quad+3 \mathrm{D}\end{array}$ & $\log \varepsilon_{N L T E}$ & $\Delta_{N L T E}$ \\
\hline & & \multicolumn{3}{|c|}{$S_{\mathrm{H}}=1$} & \multicolumn{2}{|c|}{$S_{\mathrm{H}}=0$} \\
\hline 6300 & 8.67 & 8.67 & 0.00 & 8.72 & 8.67 & 0.00 \\
\hline 6158 & 8.84 & 8.82 & -0.02 & 8.79 & 8.79 & -0.05 \\
\hline 7771 & 8.92 & 8.74 & -0.18 & 8.80 & 8.58 & -0.34 \\
\hline 7774 & 8.91 & 8.75 & -0.16 & 8.79 & 8.59 & -0.32 \\
\hline 7775 & 8.89 & 8.75 & -0.14 & 8.78 & 8.61 & -0.28 \\
\hline 8446 & 8.86 & 8.74 & -0.12 & 8.77 & 8.61 & -0.25 \\
\hline
\end{tabular}

In Fig. 3 we present the obtained non-LTE abundances as $[\mathrm{O} / \mathrm{Fe}]$ versus $[\mathrm{Fe} / \mathrm{H}]$ and for comparison non-LTE abundances from Ramirez et al. (2013). The [O/Fe] ratio increases with decreasing metallicity, in line with many previous studies. As can be seen in Fig. 3, our $[\mathrm{O} / \mathrm{Fe}]$ ratios for a few stars are higher than the data from Ramirez et al. (2013) at close metallicities. This can be due to neglecting collisions with hydrogen atoms in the latter paper. It is worth noting, that the used stellar parameters will be refined within the project.

\section{Conclusions}

A comprehensive model atom for O I produced by Przybilla et al. (2000) was updated using the best theoretical and experimental atomic data available so far. The updated model atom was tested with A-type stars. For the five stars with $T_{\text {eff }}>7250 \mathrm{~K}$ the difference in non-LTE abundances between the IR and visual lines $\Delta_{I R-V i s}$ does not exceed 0.14 dex.

From analyses of cool stars with reliable parameters (Sun and Procyon) we constrained an efficiency of collisions with hydrogen atoms and chosen the scaling factor $S_{\mathrm{H}}=1$ to the Drawin's formula. The solar mean non-LTE oxygen abundance from the O I 6300, $6158,7771-5$, and $8446 \AA$ lines is $\log \varepsilon=8.74 \pm 0.05$, when using the MAFAGS-OS solar model atmosphere and $\log \varepsilon=8.78 \pm 0.03$, and applying the $3 \mathrm{D}$ corrections from Caffau et al. (2008). Then we applied this method to the sample of dwarfs with $[\mathrm{Fe} / \mathrm{H}]$ from -1.02 to 0.32 . We obtained that the $[\mathrm{O} / \mathrm{Fe}]$ ratio increases with decreasing metallicity, in line with previous studies. This study will be continued.

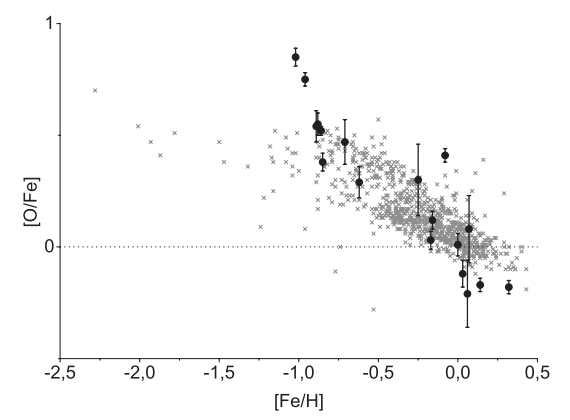

Figure 3. $[\mathrm{O} / \mathrm{Fe}]$ ratios for 18 cool dwarfs (circles). Error bars are indicated for each star. Grey crosses show the data from Ramirez et al. (2013). 
Table 4. Stellar parameters and non-LTE oxygen abundances of the sample of dwarfs.

\begin{tabular}{lcccccccc}
\hline $\mathrm{HD}$ & $T_{\text {eff }}, K$ & $\log \mathrm{g}$ & {$[\mathrm{Fe} / \mathrm{H}]$} & $\log \varepsilon_{N L T E}$ & $\sigma$ & $\Delta_{7771}$ & $N_{\text {features }}$ & $\Delta_{\text {IR }-V i s}$ \\
\hline 22484 & 5910 & 4.04 & 0.00 & 8.65 & 0.05 & -0.25 & 4 & 0.01 \\
34411 & 5560 & 4.10 & -0.08 & 8.97 & 0.03 & -0.20 & 4 & -0.01 \\
59984 & 6025 & 4.05 & -0.71 & 8.40 & 0.10 & -0.26 & 7 & -0.01 \\
45067 & 6071 & 3.97 & -0.16 & 8.60 & 0.04 & -0.29 & 4 & -0.05 \\
43318 & 6333 & 3.94 & -0.17 & 8.50 & 0.04 & -0.41 & 4 & -0.06 \\
55575 & 5987 & 4.43 & -0.25 & 8.69 & 0.16 & -0.19 & 4 & -0.11 \\
134169 & 5893 & 4.02 & -0.86 & 8.30 & 0.02 & -0.22 & 7 & 0.15 \\
23249 & 5060 & 3.98 & 0.07 & 8.79 & 0.15 & -0.13 & 3 & \\
22879 & 5952 & 4.33 & -0.85 & 8.17 & 0.04 & -0.15 & 3 & \\
30562 & 5958 & 4.10 & 0.14 & 8.61 & 0.03 & -0.21 & 3 & \\
59374 & 5847 & 4.38 & -1.02 & 8.47 & 0.04 & -0.13 & 5 & \\
45205 & 5949 & 4.13 & -0.89 & 8.29 & 0.07 & -0.20 & 3 & \\
105755 & 5818 & 3.97 & -0.96 & 8.43 & 0.03 & -0.22 & 3 & \\
19373 & 6246 & 4.30 & 0.03 & 8.55 & 0.06 & -0.25 & 3 & \\
114710 & 6089 & 4.47 & 0.06 & 8.49 & 0.15 & -0.16 & 3 & \\
82943 & 5972 & 4.37 & 0.32 & 8.78 & 0.03 & -0.23 & 3 & \\
30743 & 6453 & 4.20 & -0.62 & 8.31 & 0.07 & -0.33 & 3 & \\
49933 & 6645 & 4.16 & -0.88 & 8.31 & 0.05 & -0.33 & 3 & \\
\hline
\end{tabular}

\section{Acknowledgements}

T.S. and L.M. acknowledge a partial support from the International Astronomical Union of the participation at the IAUS 298. This work is supported by Federal agency of science and innovations (2012-1.5-12-000-1011-014/8529).

\section{References}

Allende Prieto C., Asplund M., Fabiani Bendicho P. 2004 A\&A, 423, 1109

Asplund M., Grevesse N., Sauval A. J. et al. 2004 A\&A, 417, 751

Allende Prieto C., Asplund M., Fabiani Bendicho P. 2004 A\&A, 423, 1109

Barklem P. S. 2007, A\&A, 462, 781

Butler K., Giddings J., 1985 Newsletter on Analysis of Astron. Spectra 9, Univ. of London, 723

Caffau E., Ludwig H. G., Steffen M. et al. 2008, A\&A, 488, I3, 1031

Chen Y., Zhao G., Mashonkina L. et al. 2014, this volume

Drawin H. W. 1968 Z. Physik, 211, 404

Drawin H. W. 1969 Z. Physik, 225, 483

Fossati L., Bagnulo S., Monier R. et al. 2007, A\&A 476, 911

Fossati L., Ryabchikova T., Bagnulo S. et al. 2009, A\&A 503, 945

Grupp F., Kurucz R. L., Tan K. 2009, A\&A, 503, 177

Gustafsson B., Edvardsson B., Eriksson K. et al. 2008, A\&A 486, 951

R. Kurucz http://kurucz.harvard.edu/stars/SIRIUS/ap04t9850g43k0he05y.dat

Przybilla N., Butler K., Becker S. R. et al. 2000, A\&A, 359, 1085

Ramirez I., Allende Prieto C., and Lambert D. L. 2013, ApJ,764, 78

Reetz J. 1999 Astrophysycs and Space Sience 265, 171

Shulyak D., Tsymbal V., Ryabchikova T. et al. 2004 A\&A 428, 993

Sitnova T., Mashonkina L., Ryabchikova T. 2013 Astronomy letters 39, 2, 126

\section{Discussion}

H.-G. Ludvig: Comment: I found it remarkable that you need a rather low nickel abundance to fit the [O I] $6300 \AA$ line in the Sun. Question: Do you take into account the overlap of oxygen UV trancition with the $\mathrm{L}_{\beta}$ in your NLTE calculation for oxygen?

Tatyana Sitnova: Yes, we do. 

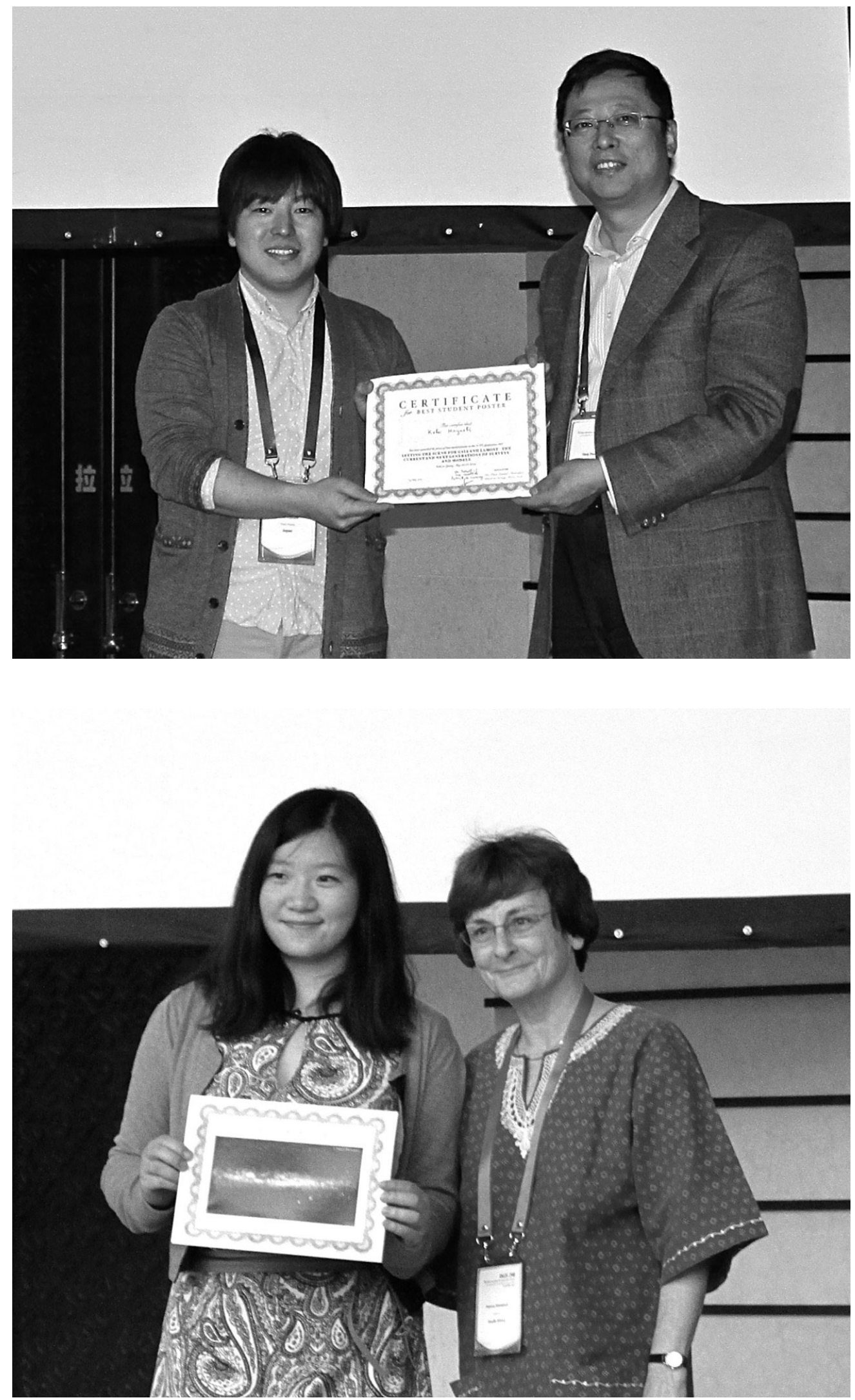